\title{
Visual Descriptors in Methods for Video Hyperlinking
}

\author{
Petra Galuščáková \\ Charles University \\ Faculty of Mathematics and Physics \\ Institute of Formal and Applied \\ Linguistics \\ Prague, Czech Republic \\ galuscakova@ufal.mff.cuni.cz \\ Jiří Matas \\ Czech Technical University in Prague \\ Faculty of Electrical Engineering \\ Department of Cybernetics \\ Center for Machine Perception \\ Prague, Czech Republic \\ matas@cmp.felk.cvut.cz
}

\author{
Michal Batko \\ Masaryk University \\ Faculty of Informatics \\ Brno, Czech Republic \\ batko@fi.muni.cz
}

\author{
David Novák \\ Masaryk University \\ Faculty of Informatics \\ Brno, Czech Republic \\ david.novak@fi.muni.cz
}

\author{
Jan Čech \\ Czech Technical University in Prague \\ Faculty of Electrical Engineering \\ Department of Cybernetics \\ Center for Machine Perception \\ Prague, Czech Republic \\ cechj@cmp.felk.cvut.cz \\ Pavel Pecina \\ Charles University \\ Faculty of Mathematics and Physics \\ Institute of Formal and Applied \\ Linguistics \\ Prague, Czech Republic \\ pecina@ufal.mff.cuni.cz
}

\begin{abstract}
In this paper, we survey different state-of-the-art visual processing methods and utilize them in hyperlinking. Visual information, calculated using Features Signatures, SIMILE descriptors and convolutional neural networks ( $\mathrm{CNN})$, is utilized as similarity between video frames and used to find similar faces, objects and setting. Visual concepts in frames are also automatically recognized and textual output of the recognition is combined with search based on subtitles and transcripts. All presented experiments were performed in the Search and Hyperlinking 2014 MediaEval task and Video Hyperlinking 2015 TRECVid task.
\end{abstract}

\section{KEYWORDS}

Video retrieval, Hyperlinking, Information retrieval, Image processing

\section{ACM Reference format:}

Petra Galuščáková, Michal Batko, Jan Čech, Jiří Matas, David Novák, and Pavel Pecina. 2017. Visual Descriptors in Methods for Video Hyperlinking. In Proceedings of ICMR '17, Bucharest, Romania, June 06-09, 2017, 7 pages. https://doi.org/10.1145/3078971.3079026

\section{INTRODUCTION}

The aim of this paper is to review methods for visual data processing and test them on hyperlinking problem. Some methods have already been utilized and described in our former work. Feature Signatures (Section 2.2) were described in the MediaEval 2014 report [17] and TRECVid results (Section 3) were described in the TRECVid report [19]. In addition to these results, we also closely analyze results achieved by Feature Signatures, show why they improve

\section{c) (1) (ㅇ)}

This work is licensed under a Creative Commons Attribution-NonCommercial-ShareAlike International 4.0 License.

ICMR '17, fune 06-09, 2017, Bucharest, Romania

(c) 2017 Copyright held by the owner/author(s).

ACM ISBN 978-1-4503-4701-3/17/06.

https://doi.org/10.1145/3078971.3079026 hyperlinking based on textual content (Section 2.3) and compare them to state-of-the-art CNN descriptors (Section 2.5). Moreover, we also present novel approaches to combination text-based retrieval with described visual descriptors (Section 2.4) and with detected visual concepts (Section 2.6). We also describe utilization of face descriptors (Section 2.7), which have not been used in hyperlinking before, according to our best knowledge.

Hyperlink is defined as "an electronic link providing direct access from one distinctively marked place in a hypertext or hypermedia document to another in the same or a different document" [31]. The source marked place of the link (e.g. relevant text, relevant part of an image or video) is called the anchor. Hyperlinking task focuses on automatic linking of the anchors to related targets. In this paper, we focus on hyperlinking in video collections. Both the anchors (or queries in our case) and targets are segments of video recording and they are defined by the video recording, beginning playback time and end playback time [36].

\subsection{Evaluation Benchmarks}

Hyperlinking of video data was also part of Search and Hyperlinking task at the 2014 MediaEval Multimedia Benchmark [15] and Video Hyperlinking task at 2015 TRECVid evaluation workshop [38]. Both tasks tested submitted systems using the same collection of TV broadcast programmes provided by BBC. The development set of the collection consisted of BBC TV programmes broadcast between 01.04 .2008 and 11.05.2008, and the test set consisted of programmes broadcast between 12.05.2008 and 31.07.2008. Among other things, the collection contained news (e.g. BBC Breakfast, BBC News at Ten), documentaries (e.g. The Life of Mammals, Wild China), serials (e.g. EastEnders, Two Pints of Lager and a Packet of Crisps), entertainment programmes (e.g. Top Gear, Hard Sell), quiz shows (e.g. Eggheads, The Weakest Link), cookery shows (e.g. Saturday Kitchen, Ready Steady Cook), sport events (e.g. football matches, horse races), and children's programmes (e.g. Big Barn Farm, Nina and the Neurons). The collection also contained concerts (e.g. Radio 1's Big Weekend, Glastonbury The Best Bits) and music programmes (e.g. Mad about Music, Later... with fools Holland). Thus the range of 
topics of the videos was unrestricted and the collection contained many speakers, settings, accents, genres, and formality types. Apart from videos, the collection was also published with subtitles and three automatic transcripts provided by LIMSI [26], LIUM [43] and NST-Sheffield [27], manually entered metadata, automatically detected shots, a list of stable keyframes, prosodic features [16], and visual concepts $[8,46]$.

The set of query segments was in both tasks collected by hired participants of a user survey. In the 2014 MediaEval task, the focus was on a home user scenario. Participants searched the collection using a text-based search and watched some retrieved segments. Then, they marked anchors within these segments. Participants were also asked to state what kind of links would they like to find using marked segments. This information was further available in the evaluation but it was hidden to the task participants. 30 out of 36 collected queries were used for evaluation.

The 2015 Video Hyperlinking task was focused on media professionals and content creators. The anchors were created by journalist students and media professionals who were asked to locate segments which they would like to enrich by hyperlinks if they were content producers. Totally, 100 queries out of 135 collected were used for evaluation.

\subsection{Hyperlinking Approaches}

Ordelman et al. [36] state that the main difference between video hyperlinking task and other related problems such as video recommendation or near-duplicate detection is that video hyperlinking focuses on "'give me more information about this anchor' instead of 'give me more based on this anchor or entity'". Thus the attention is focused on semantic similarity between the anchoring and target segments.

A full range of methods had been applied in the hyperlinking. In order to find the relevant segment, pre-segmentation of the videos is the dominant approach. Researchers use fixed length segments measured by time $[10,11,33]$ or number of included words [29], sentence boundaries [9], shots [30, 41], visual similarity between frames [45] and boundaries defined by the TextTilling $[3,39]$ segmentation algorithm. However, some researches localize directly the jump-in points without any pre-segmentation. For example García et al. [20] put jump-in point to the place which maximizes their similarity function based on shared words and WordNet distances between words. Similarly, Preston et al. [42] represent each video by a probability density function and the points with the highest probabilities are the jump-in points.

Methods also differ in types of modalities which are used in the retrieval. Some of the authors only use textual information from transcripts and subtitles [20,29]. Textual information can also be enriched by synonyms [41] or named entities [10,34]. Textual query expanded by connected entities from Freebase, geographical entities from GeoNames, synonyms from Wordnet, and DBpedia words were used by Lokaj et al. [30]. Word vectors [32] can also be used as word representations [39]. Textual information is often combined with the visual information. The researches mostly employ visual concepts [28, 45, 49]. Şimon et al. [13] use bi-lingual LDA to create a probabilistic translation model between lexical transcripts and visual concepts. The results acquired by the text-based retrieval

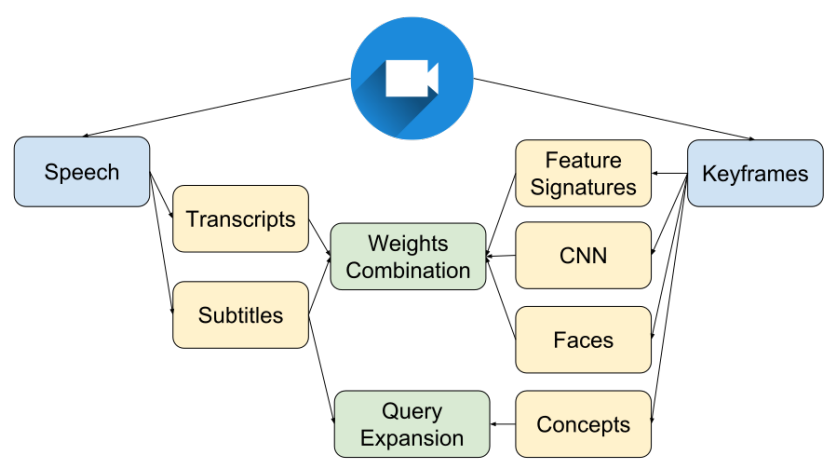

Figure 1: Visual methods overview.

using subtitles and transcripts can also be re-ranked using SIFT descriptors [30]. On the other hand, the approach by Werner and Ward [47] is purely based on the prosodic information. However, improvement achieved using multiple modalities is not definite. Even though, that some researches report an improvement using a combination of modalities, the best scoring approach at the 2015 TRECVid [11] only uses textual data.

The remainder of the paper is organized as follows. First, methods for calculating similarity between frames tested in MediaEval 2014 [17] will be described. Retrieval which utilizes Visual Similarity using Feature Signatures, which bring information about frame setting, will be compared with retrieval methods based purely on subtitles and transcripts. Results will be analyzed in respect to the TV broadcast data. In the following experiments, Feature Signatures will be compared with deep-learning based methods and face similarity algorithms will be utilized. Finally, the most promising approaches will be compared in the 2015 TRECVid evaluation benchmark.

\section{VISUAL DESCRIPTORS IN MEDIAEVAL 2014}

This section describes different visual descriptors (Feature Signatures, $\mathrm{CNN}$ and face descriptors) and different ways how to combine them with text-based retrieval (linear combination of weights and query expansion in which words from query are used together with detected concepts). Experiments with these descriptors were performed in MediaEval 2014 Benchmark and after the Benchmark on the same dataset. Overview of described methods is displayed in Figure 1.

\subsection{Text-based Retrieval}

In order to easily retrieve relevant video passages, recordings were divided into 50-second long segments. A new segment was created each 10 seconds - created segments can thus overlap. Text lying within the segment boundaries was then indexed. Similarly, we created a textual query from a query segment by utilizing all words in the subtitles lying within the query segment. Text-based retrieval can then be applied to the collection. In our experiments, we used the Terrier IR Framework [37] and its implementation of the Hiemstra Language Model [21] for retrieval, with its parameter set to 0.35 . We used Porter stemmer and Terrier's stopwords list. In all experiments, we post-filtered retrieved segments and removed 
segments which partially overlapped with the query segment and also possibly with another higher ranked retrieved segment (further called removal of overlapping or $R O$ filtering).

Manually created metadata available in the collection were utilized to further enrich the retrieval - title, episode title, description, short episode synopsis, service name, and program variant of the programmes were used specifically. Each segment was concatenated with the metadata of the corresponding full recording. Additionally, each query segment was concatenated with the metadata of the corresponding full recording. As the context of the query segment proved to be helpful in the former experiments, 200 seconds before the beginning of the query segment and 200 seconds after the end of the query segment were also utilized. The length of the context was tuned on the development data.

\section{$2.2 \quad$ Feature Signatures}

In order to represent video frames, Feature Signatures that approximate distribution of color and texture in the image can be used. Unlike modern CNN descriptors excellent in recognizing specific objects, this traditional descriptor can be used to identify keyframes with a similar background and setting. Feature Signatures have already been used in a known item search tasks [12]. The authors have created an efficient and effective sketch-based retrieval system $[4,5]$, where each keyframe is represented by a Feature Signature and users can query the database of Feature Signatures using simple signature-based sketches.

Visual Similarity was calculated between the query segment and each data segment. Distance was calculated between each keyframe in the query segment and each keyframe in data. Distance between segments was then calculated as a minimal similarity (1-distance) between keyframes in the query and data segments. Finally, Visual Similarity of the segments was linearly combined with their Textual Similarity acquired from the text-based retrieval:

$$
\begin{aligned}
& \text { FinalScore }(\text { segment } / \text { query })=\text { Score }(\text { segment } / \text { query }) \\
& + \text { Weight } * \text { VisualSimilarity }(\text { segment } / \text { query }),
\end{aligned}
$$

and the retrieved segments were scored according to the Final Score. The Weight was tuned on the development data and it prefers textual information.

Position-color-texture Feature Signatures [23, 24, 44] were utilized to approximate a distribution of color and texture in each keyframe. This descriptor can be utilized in image retrieval tasks, where color and texture is meaningful for retrieval. Formally, given a feature space $\mathbb{F}$, the Feature Signature $S^{o}$ of a multimedia object $o$ is defined as a set of tuples $\left\{\left\langle r_{i}^{o}, w_{i}^{o}\right\rangle\right\}_{i=1}^{n}$ from $\mathbb{F} \times \mathbb{R}^{+}$, consisting of representatives $r_{i}^{o} \in \mathbb{F}$ and weights $w_{i}^{o} \in \mathbb{R}^{+}$. Image is then represented by a sequence of Feature Signatures which describe color of image regions. Example of image represented by Feature Signatures is displayed in Figure 2.

The distance between Features Signatures was calculated using the Signature Quadratic Form Distance [2]. Keyframe distances were calculated and provided by Department of Software Engineering at Charles University. Employing Feature Signatures enables visual similarity to work exceptionally well in detecting similar settings and backgrounds. This is particularly important in working
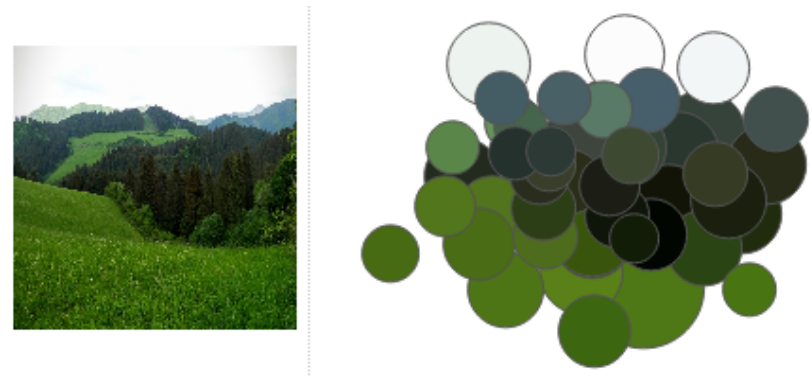

Figure 2: Representation of image using Feature Signatures.

with TV collections, in which a similar background occurs throughout the series. However Feature Signatures can fail to detect some details in keyframes, e.g. it is not possible recognize a particular person.

\subsection{MediaEval 2014 Results}

The task was evaluated manually using crowd-sourcing methods and MAP-bin and MAP-tol evaluation measures [1]. Both these measures are adaptations of the MAP measure proposed for evaluation of video content retrieval to allow a segment retrieved near the relevant segment (but not necessarily overlapping it) to also be marked as relevant.

Comparison of results with utilization of Visual Similarity using Feature Signatures and using purely transcripts and subtitles is displayed in Table 1 . The best results for both measures were achieved when the Visual Similarity was employed. The highest MAP-bin score was achieved on the subtitles when the overlapping segments were preserved. In general, differences between subtitles and automatic transcripts were minor, especially when additional visual information was used. The highest MAP-tol score was surprisingly achieved on the LIMSI transcripts when overlapping segments were filtered out. This may be caused by a precisely given timing of each word in the LIUM transcript. The timing of each word in the subtitles was approximated, based on the time of the beginning and the end of the utterance. In the case of the 15-second long tolerance windows, this approximation could decrease the score.

\begin{tabular}{ccccc} 
Transcript & Weights & RO & MAP-bin & MAP-tol \\
\hline Subtitles & None & Yes & 0.2689 & 0.2465 \\
Subtitles & Visual & No & $\mathbf{0 . 3 0 8 0}$ & 0.0996 \\
Subtitles & Visual & Yes & 0.2724 & $\mathbf{0 . 2 5 8 0}$ \\
\hline LIMSI & None & Yes & 0.2659 & 0.2297 \\
LIMSI & Visual & No & $\mathbf{0 . 3 0 4 2}$ & 0.0950 \\
LIMSI & Visual & Yes & 0.2692 & $\mathbf{0 . 2 4 1 4}$ \\
\hline LIUM & None & Yes & 0.2593 & 0.2547 \\
LIUM & Visual & No & $\mathbf{0 . 2 8 8 0}$ & 0.0993 \\
LIUM & Visual & Yes & 0.2622 & $\mathbf{0 . 2 6 3 2}$ \\
\hline NST-Sheffield & None & Yes & 0.2611 & 0.2237 \\
NST-Sheffield & Visual & No & $\mathbf{0 . 2 9 4 9}$ & 0.0914 \\
NST-Sheffield & Visual & Yes & 0.2655 & $\mathbf{0 . 2 4 4 0}$
\end{tabular}

Table 1: Results with and without visual similarity utilization for different transcripts with and without RO filtering. Best results for each transcript are highlighted. 
Visual Similarity calculated using Feature Signatures proved to be very helpful in the task. Even though the improvement in terms of MAP-bin score was minor, Feature Signatures improved the results consistently on all transcripts in both measures. Low MAP-tol scores achieved when overlapping segments were not filtered were caused by that MAP-tol measure takes into account each relevant segment only once. Overlapping retrieved segments thus lowered the score. Presented systems also achieved the overall highest results in the Benchmark [14].

Three examples in which Feature Signatures improved the retrieval quality are displayed in Figure 3. Color-position-based Feature Signatures work well for type of queries similar to Query 2. Even though that query and target keyframes are not necessarily from the same TV series or program, they are similar in terms of what color occurs in which part of the image. Similarly, the results in Query 11 are improved thanks to the retrieval of the same setting. In the Query 26, the only top relevant retrieved segment was the segment before the beginning of the query segment. This segment was retrieved thanks to its high Visual Similarity with the query segment. It did not occur among the top results retrieved using the text-based similarity only.

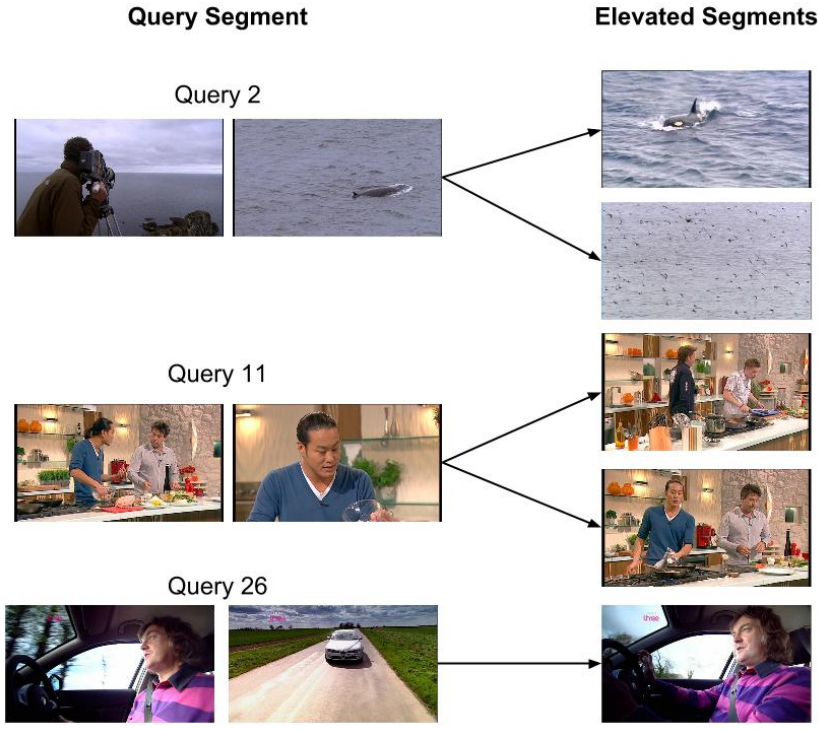

Figure 3: Examples in which employment of the Visual Similarity increased the retrieval quality.

Several keyframes of the query segments in which Visual Similarity decreased the results are in Figure 4. These keyframes display mainly persons and they do not contain any specific content or background. Therefore, even an optimal Visual Similarity calculation is hardly expected to improve the results in these cases.

\subsection{Combination with Text-based Retrieval}

In order to combine text-based retrieval with Visual Similarity between keyframes, maximum similarity between any keyframe from the query segment and any keyframe from the target segment was used in the presented experiments. However, this strategy is not
Query 19
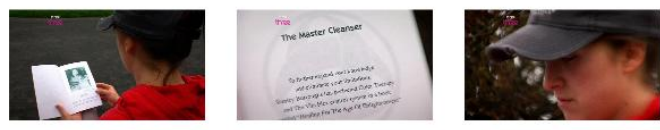

Query 21
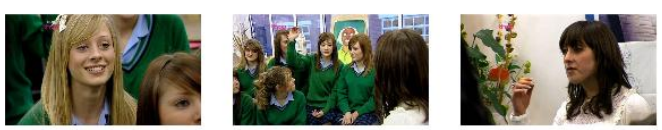

Figure 4: Examples in which employment of the Visual Similarity decreased the retrieval quality.

optimal as single keyframes can be similar to each other accidentally, e.g. both segments can contain black keyframes. Several other strategies were thus tested in follow-up experiments. Visual Similarity was also calculated as a sum over two or three most similar keyframes. Moreover, these two or three keyframes can be selected from segments with or without repetition - one keyframe thus can be taken into account either once or multiple times.

Additionally, in the case of calculating similarity using two keyframes, only keyframes which were similar enough to their adjacent keyframes were taken into account. This simulated that selected keyframes are specific representatives of the segments. Similarly, only keyframes which differed enough from the adjacent keyframes were taken into account, which simulated that selected keyframes are distinctive in comparison with their surrounding. In addition to maximum similarity, we also calculated average similarity between all pairs of keyframes in query and target segments.

\begin{tabular}{cccc} 
Transcript & Keyframes & MAP-bin & MAP-tol \\
\hline Subtitles & None & 0.1463 & 0.1335 \\
Subtitles & Max 1 & 0.1556 & 0.1389 \\
Subtitles & Max 2 & 0.1622 & $\mathbf{0 . 1 4 4 5}$ \\
Subtitles & Max 3 & $\mathbf{0 . 1 6 2 3}$ & 0.1423 \\
\hline
\end{tabular}

Table 2: Results for Visual Similarity calculated using different number of keyframes. Best results are highlighted.

Each of these approaches increased both MAP-bin and MAPtol scores. Highest achieved scores are tabulated in Table $2^{1}$. The approach which uses similarity between two keyframes (each one was selected from the segment without a repetition) achieved the most promising results. For three most similar keyframes, the MAPbin score was even slightly higher but the improvement for the MAP-tol slightly dropped.

\subsection{Object Recognition}

In contrast to position-color-texture-based Feature Signatures, modern deep convolutional neural networks work particularly well for recognition and classification of object in the image [22]. Similarity between keyframes was thus also calculated using a CNN visual features calculated as the output from the last hidden layer fc7 of AlexNet [22]. These 4096-dimensional vectors were organized for efficient similarity search by a distance-based index [35] and this

\footnotetext{
${ }^{1}$ Even though, the experiments were performed on the same data as those in the previous section, the baseline system in these experiments uses 60 -second long segments instead of 50 and no metadata and context are used.
} 
system provided the similarity scores. Calculated object similarity was linearly combined with text-based similarity in the same way as similarity calculated using Feature Signatures. Similarly to Feature Signatures, $\mathrm{CNN}$ improved purely text-based retrieval but the improvement was smaller than in the case of the Feature Signatures and CNN were thus not applied on the 2015 TRECVid test data.

Both Visual Similarity calculation models were also combined. First, they were combined linearly with the output of text-based retrieval similarly as in the previous experiments. In order to acquire comparable scores, maxmin and mean normalization was applied to both visual similarity scores and both weights were jointly tuned. However, this approach did not outperform combination of textbased retrieval and Feature Signatures.

Scores were also combined using re-ranking. CNN were first applied and combined with text-based search in order to retrieve similar objects. Top retrieved results (10, 20, 50 and 100 items) then were re-ranked using Feature Signatures in order to sort target keyframes according to their "overall" similarity with the query keyframe. Although, this approach achieved a minor improvement, it again did not outperform results achieved by basic linear combination of text and Feature Signatures.

\subsection{Concept Detection}

Convolutional networks were also used to acquire textual descriptions of the concepts depicted in the keyframes. Concepts were created by image annotation system used in ImageCLEF 2014 Task [7]. This system first retrieves images similar to a given query image, or keyframe in our case, from annotated Profiset [6] using CNN. The word descriptions of the most similar images are then used for text analysis where the word semantic relationships are exploited to create a description of the query image. The description was created for each keyframe as a list of concepts (e.g. people, indoors, young, two, canadian, plant, macro) with a confidence score assigned to each concept.

\begin{tabular}{cccccc} 
Transcript & Concepts & Max Conc. & RO & MAP-bin & MAP-tol \\
\hline Subtitles & No & - & No & 0.2333 & 0.1375 \\
Subtitles & Yes & - & No & $\mathbf{0 . 2 4 1 4}$ & 0.1603 \\
Subtitles & Yes & 5 & No & 0.2368 & $\mathbf{0 . 1 6 3 8}$ \\
\hline
\end{tabular}

Table 3: Query expansion by visual concepts with and without restriction of the maximum number of concept occurrences. Concepts are weighted by their confidence scores in both cases. Best results are highlighted.

Detected concepts were appended to the created text-based queries. When we used all detected concepts of all query keyframes, both the scores have decreased since the concepts then contained too much noise. Using only concepts with higher confidence scores and concepts with restricted number of occurrences (occurring up to two or three times in the query segment) allowed to increase these results but the scores were still lower than the baseline. However, assigning concept confidence scores as query term weights increased both the scores. Highest MAP-tol result was achieved using combination of concept term weighting and restriction of number of occurrences of each concept in query (up to six occurrences). The results achieved for query expansion using concepts are shown in Table 3.

\subsection{Faces recognition}

In addition to recognition of settings and particular objects, we also experimented with face recognition. Faces were detected by a commercial multi-view face detector ${ }^{2}$. Each face was geometrically aligned with a canonical pose by automatically extracted facial landmarks. Then a compact vectorial SIMILE descriptor [25] was calculated. A set of face descriptors representing person identities were thus available for each keyframe in which faces were detected. Faces were then compared by L2 distance on calculated descriptors.

In this way, it was possible to find occurrences of persons from query keyframes in the rest of the data. Detected similarity was first linearly combined with text-based similarity in the same way as Visual Similarity. This combination very slightly increased the MAP-bin score from 0.1463 to 0.1473 but MAP-tol score dropped from 0.1335 to 0.1322 . Additionally, faces similarity was used for reranking top 1000 text-based retrieval results. In this case, both scores were improved. MAP-bin increased from 0.2051 to 0.2088 in the case when only faces with similarity score higher than a given threshold were used. MAP-tol score increased from 0.1162 to 0.1281 when all available faces were used. Due to unstable improvement achieved on the training data, this approach was not applied on the test data. We expect that employing recent deep net descriptors that are more powerful, e.g. [40], would further improve the performance.

\section{TRECVID 2015 METHODS COMPARISON}

The Search and Hyperlinking 2014 MediaEval task and Video Hyperlinking 2015 TRECVid task shared the same dataset but differed in the set of queries used for evaluation. Test set used for evaluation of the Search and Hyperlinking task was available for development purposes in the Video Hyperlinking task. Therefore, approaches which achieved the most promising results in the MediaEval 2014 experiments and in described follow-up experiments were submitted to the TRECVid 2015 Video Hyperlinking Task. In this section, we present the submitted systems.

\subsection{Baseline run}

Baseline is similar to the system described in Section 2.1 but there are some minor differences. Recordings were segmented into 60second passages, only a title, a description and broadcast channel name were used as metadata information and context of $20 \mathrm{sec}-$ onds was used. No automatic transcripts, only subtitles were used in the submitted runs. As the BBC collection contains numerous music programmes, the query segments were also expanded by audio information contained in each segment [18]. Sub-segments of each segment then were submitted to the Doreso service ${ }^{3}$ for music identification which uses a fingerprinting technique. If the sub-segment contains any music, the song title and the artist are retrieved and this information is concatenated with the query segment. Music was detected in 10 out of 30 training queries but only in 7 out of 135 test queries. Each query was also further expanded

\footnotetext{
${ }^{2}$ Eyedea Recognition Ltd. http://www.eyedea.cz/

${ }^{3}$ http://developer.doreso.com
} 


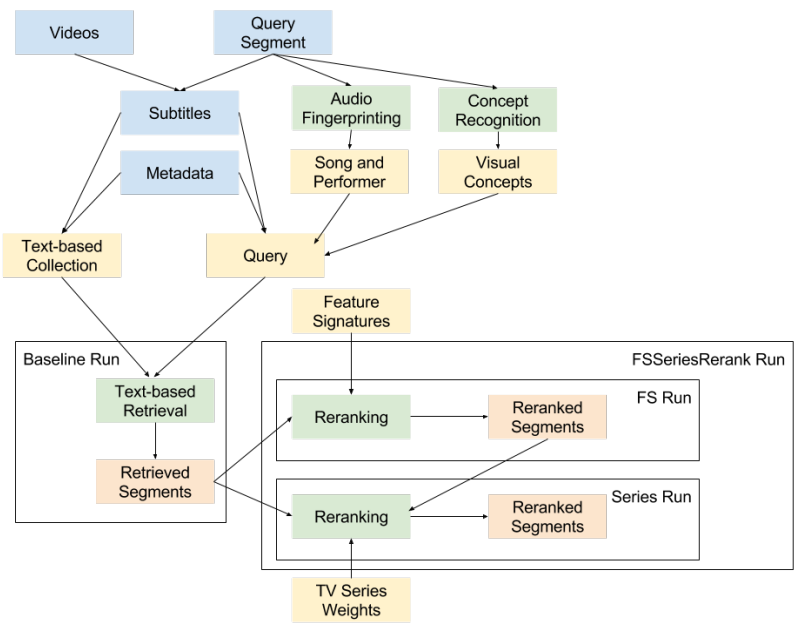

Figure 5: Retrieval processing system - strategy diagram.

by visual concepts contained in the source video segments which were weighted by confidence scores and only used if they occurred less than 7 times in segment as described in Section 2.6.

\subsection{Tuning}

The Series run achieved the highest improvement on the training data. It combines the Baseline run with information on whether the videos came from the same TV series. This information was used to pre-calculate a "series weight" for each video which was set to 0.13 if the query video and data video were from the same TV series; otherwise the weight was set to -0.15 . These weights were tuned on the training data. Weights were linearly combined with the top 1000 retrieved results.

The FS run is similar to the highest ranked run submitted to the MediaEval 2014 Benchmark. In this run, the Baseline was expanded by the Visual Similarity between the query segment and data segments calculated using Feature Signatures as described in Section 2.2. Visual Similarity was calculated using two most similar keyframes as described in Section 2.4.

Finally, the FSSeriesRerank run is a combination of the FS and the Series runs. The top 1000 results returned by the FS system were linearly combined with the same weights as those used in the Series run and re-ranked accordingly.

\subsection{Results}

The strategy diagram of retrieval approaches is displayed in Figure 5. A performance comparison is reported in Table 4.

The best results are again achieved using Feature Signatures as the FS run outperforms the Baseline run. Even though that this improvement is not significant ${ }^{4}$, it is in line with our previous experiments and it confirms that employment of Visual Similarity consistently improves text-based retrieval. Weighting using information about the same TV series did not outperform the Baseline run in any case; the Baseline run is significantly better than both

\footnotetext{
${ }^{4}$ Wilcoxon signed rank test $[48]$
}

\begin{tabular}{cccc} 
Run Num. & Run Name & MAP-bin & MAP-tol \\
\hline 1 & Series & 0.1443 & 0.1131 \\
2 & FSSeriesRerank & 0.1094 & 0.0838 \\
3 & FS & $\mathbf{0 . 1 5 6 0}$ & $\mathbf{0 . 1 2 3 4}$ \\
4 & Baseline & 0.1536 & 0.1214 \\
\hline
\end{tabular}

Table 4: Performance comparison of results submitted to the Video Hyperlinking Task. Best results for each measure are highlighted.

Series and FSSeriesRerank runs, despite high improvement of reranking based on TV series achieved on the training data.

\section{CONCLUSIONS}

In the paper, we complexly reviewed image processing methods and tested them on the video hyperlinking task. We presented a range of image processing methods (Feature Signatures, CNN, concept detection and faces recognition) and experimented with their combination with text-based retrieval using transcripts and subtitles. All of these methods achieved improvement on the training data, comparing with text-based retrieval. Most promising methods which use Feature Signatures and detected concepts were also used in MediaEval and TRECVid benchmark experiments in which we confirmed that appropriate visual information improves results achieved using text-based retrieval. Even though that the improvement achieved by Feature Signatures was not significant, it was constantly better than text-based retrieval.

\section{ACKNOWLEDGMENTS}

The authors would like to thank to Jakub Lokoč and Martin Kruliš from the SIRET Group, Department of Software Engineering at Charles University for providing data. This research is supported by the Czech Science Foundation, grant number P103/12/G084.

\section{REFERENCES}

[1] Robin Aly, Maria Eskevich, Roeland Ordelman, and Gareth J. F. Jones. 2013. Adapting Binary Information Retrieval Evaluation Metrics for Segment-based Retrieval Tasks. CoRR abs/1312.1913 (2013).

[2] Christian Beecks, Merih Seran Uysal, and Thomas Seidl. 2010. Signature Quadratic Form Distance. In Proc. of CIVR. Xi'an, China, 438-445.

[3] Chidansh A. Bhatt, Nikolaos Pappas, Maryam Habibi, and Andrei PopescuBelis. 2014. Multimodal Reranking of Content-based Recommendations for Hyperlinking Video Snippets. In Proc. of ICMR. Glasgow, UK, 225-232.

[4] Adam Blažek, Jakub Lokoč, Filip Matzner, and Tomáš Skopal. 2015. Enhanced Signature-Based Video Browser. In Proc. of MMM (Lecture Notes in Computer Science), Vol. 8936. Sydney, Australia, 243-248.

[5] Adam Blažek, Jakub Lokoč, and Tomáš Skopal. 2014. Video Retrieval with Feature Signature Sketches. In Proc of SISAP (Lecture Notes in Computer Science), Vol. 8821. Los Cabos, Mexico, 25-36.

[6] Petra Budikova, Michal Batko, and Pavel Zezula. 2011. Evaluation Platform for Content-based Image Retrieval Systems. In International Conference on Theory and Practice of Digital Libraries (TPDL 2011). 130-142.

[7] Petra Budíková, Jan Botorek, Michal Batko, and Pavel Zezula. 2014. DISA at ImageCLEF 2014: The Search-based Solution for Scalable Image Annotation. In Proc. of CLEF. Sheffield, UK, 360-371.

[8] Ken Chatfield, Relja Arandjelović, Omkar Parkhi, and Andrew Zisserman. 2015. On-the-fly Learning for Visual Search of Large-scale Image and Video Datasets. International fournal of Multimedia Information Retrieval (2015), 1-19.

[9] Shu Chen, Keith Curtis, David N. Racca, Liting Zhou, Gareth J.F. Jones, and Noel E. O'Connor. 2015. DCU ADAPT @ TRECVid 2015: Video Hyperlinking Task. In Proc. of TRECVID. Gaithersburg, MD, USA.

[10] Shu Chen, Gareth J. F. Jones, and Noel E. O'Connor. 2013. DCU Linking Runs at MediaEval 2013: Search and Hyperlinking Task. In Proc. of MediaEval. Barcelona, Spain. 
[11] Zhiyong Cheng, Xuanchong Li, Jialie Shen, and Alexander G. Hauptmann. 2015 CMU-SMU@TRECVID 2015: Video Hyperlinking. In Proc. of TRECVID. Gaithersburg, MD, USA.

[12] Claudiu Cobârzan, Klaus Schoeffmann, Werner Bailer, Wolfgang Hürst, Adam Blažek, Jakub Lokoč, Stefanos Vrochidis, Kai Uwe Barthel, and Luca Rossetto. 2017. Interactive Video Search Tools: A Detailed Analysis of the Video Browser Showdown 2015. Multimedia Tools and Applications 76, 4 (2017), 5539-5571.

[13] Anca-Roxana Simon, Ronan Sicre, Rémi Bois, Guillaume Gravier, Pascale Sébillot, and Emmanuel Morin. 2015. IRISA at TrecVid2015: Leveraging Multimodal LDA for Video Hyperlinking. In Proc. of TRECVID. Gaithersburg, MD, USA.

[14] Maria Eskevich, David N. Racca Aly, Robin and, Roeland Ordelman, Shu Chen, and Jones Gareth J.F. 2014. Search and Hyperlinking 2014 Overview. (2014). http://www.slideshare.net/mariaeskevich/ search-and-hyperlinking-me14sh-task-overviewmero

[15] Maria Eskevich, Robin Aly, David N. Racca, Roeland Ordelman, Shu Chen, and Gareth J.F. Jones. 2014. The Search and Hyperlinking Task at MediaEval 2014. In Proc. of MediaEval. Barcelona, Spain.

[16] Florian Eyben, Felix Weninger, Florian Gross, and Björn Schuller. 2013. Recent Developments in openSMILE, the Munich Open-source Multimedia Feature Extractor. In Proc. of ACMMM. Barcelona, Spain, 835-838.

[17] Petra Galuščáková, Martin Kruliš, Jakub Lokoč, and Pavel Pecina. 2014. CUNI at MediaEval 2014 Search and Hyperlinking Task: Visual and Prosodic Features in Hyperlinking. In Proc. of MediaEval. Barcelona, Spain.

[18] Petra Galuščáková and Pavel Pecina. 2015. Audio Information for Hyperlinking of TV Content. In Proc. of SLAM. Brisbane, Australia, 27-30.

[19] Petra Galuščáková, Michal Batko, Martin Kruliš, Jakub Lokoč, David Novák, and Pavel Pecina. 2015. CUNI at TRECVID 2015 Video Hyperlinking Task. In Proc. of TRECVID. Gaithersburg, MD, USA.

[20] Fernando García, Emilio Sanchis, Marcos Calvo, Ferran Pla, and Lluís-F. Hurtado 2013. ELiRF at MediaEval 2013: Similar Segments in Social Speech Task. In Proc. of MediaEval. Barcelona, Spain.

[21] Djoerd Hiemstra. 2001. Using Language Models for Information Retrieval. Ph.D. Dissertation. University of Twente, Enschede, Netherlands.

[22] Alex Krizhevsky, Ilya Sutskever, and Geoffrey E. Hinton. 2012. ImageNet Classification with Deep Convolutional Neural Networks. In Advances in Neural Information Processing Systems 25. 1097-1105.

[23] Martin Kruliš, Tomáš Skopal, Jakub Lokoč, and Christian Beecks. 2012. Combining CPU and GPU Architectures for Fast Similarity Search. Distributed and Parallel Databases 30, 3-4 (2012), 179-207.

[24] Martin Kruliš, Jakub Lokoč, and Tomáš Skopal. 2016. Efficient Extraction of Clustering-based Feature Signatures Using GPU Architectures. Multimedia Tools and Applications 75, 13 (July 2016), 8071-8103.

[25] Neeraj Kumar, Alexander C. Berg, Peter N. Belhumeur, and Shree K. Nayar. 2009. Attribute and Simile Classifiers for Face Verification. In Proc. of ICCV. Kyoto, Japan, 365-372.

[26] Lori Lamel and Jean-Luc Gauvain. 2008. Speech Processing for Audio Indexing In Proc. of GoTAL 2008, Advances in NLP. Gothenburg, Sweden, 4-15.

[27] P. Lanchantin, P.-J. Bell, M.-J.-F. Gales, T. Hain, X. Liu, Y. Long, J. Quinnell, S Renals, O. Saz, M.-S. Seigel, P Swietojanski, and P.-C. Woodland. 2013. Automatic Transcription of Multi-genre Media Archives. In Proc. of SLAM Workshop. Marseille, France, 26-31.

[28] H.A. Le, B. Huet Q.M. Bui and, B. Červenková, J. Bouchner, E. Apostolidis, F. Markatopoulou, A. Pournaras, V. Mezaris, D. Stein, S. Eickeler, and M Stadtschnitzer. 2014. LinkedTV at MediaEval 2014 Search and Hyperlinking Task. In Proc. of MediaEval. Barcelona, Spain.

[29] Gina-Anne Levow. 2013. UWCL at MediaEval 2013: Similar Segments in Social Speech Task. In Proc. of MediaEval. Barcelona, Spain.

[30] Michał Lokaj, Harald Stiegler, and Werner Bailer. 2013. TOSCA-MP at Search and Hyperlinking of Television Content Task. In Proc. of MediaEval. Barcelona Spain.

[31] Merriam-Webster Online. 2009. Merriam-Webster Online Dictionary. (2009). http://www.merriam-webster.com

[32] Tomas Mikolov, Scott Wen tau Yih, and Geoffrey Zweig. 2013. Linguistic Regularities in Continuous Space Word Representations. In Proc. of HLT NAACL. Atlanta, GA, USA.

[33] Usman Niaz, Bernard Merialdo, Claudiu Tanase, Maria Eskevich, and Benoit Huet. 2015. EURECOM at TrecVid 2015: Semantic Indexing and Video Hyperlinking Tasks. In Proc. of TRECVID 2015. Gaithersburg, MD, USA

[34] Tom De Nies, Wesley De Neve, Erik Mannens, and Rik Van de Walle. 2013. Ghen University-iMinds at MediaEval 2013: An Unsupervised Named Entity-based Similarity Measure for Search and Hyperlinking. In Proc. of MediaEval. Barcelona Spain.

[35] David Novák, Michal Batko, and Pavel Zezula. 2015. Large-scale Image Retrieval using Neural Net Descriptors. In Proc. of SIGIR '15. Santiago, Chile.

[36] Roeland Ordelman, Maria Eskevich, Robin Aly, Benoit Huet, and Gareth J. F. Jones 2015. Defining and Evaluating Video Hyperlinking for Navigating Multimedia Archives. In Proc. of WWW (Companion Volume). Florence, Italy, 727-732.
[37] I. Ounis, G. Amati, V. Plachouras, B. He, C. Macdonald, and C. Lioma. 2006. Terrier: A High Performance and Scalable Information Retrieval Platform. In Proc. of SIGIR Workshop on Open Source Information Retrieval. Seattle, WA, USA, $18-25$.

[38] Paul Over, George Awad, Martial Michel, Jonathan Fiscus, Wessel Kraaij, Alan F. Smeaton, Georges Quéenot, and Roeland Ordelman. 2015. TRECVID 2015 - An Overview of the Goals, Tasks, Data, Evaluation Mechanisms and Metrics. In Proc. of TRECVID. Gaithersburg, MD, USA.

[39] Lei Pang and Chong-Wah Ngo. 2015. VIREO @ TRECVID 2015: Video Hyperlinking (LNK). In Proc. of TRECVID 2015. Gaithersburg, MD, USA

[40] Omkar M. Parkhi, Andrea Vedaldi, and Andrew Zisserman. 2015. Deep Face Recognition. In Proc. of BMVC. Swansea, UK, 41.1-41.12.

[41] Zsombor Paróczi, Bálint Fodor, and Gábor Szücs. 2014. DCLab at MediaEval2014 Search and Hyperlinking Task. In Proc. of MediaEval. Barcelona, Spain.

[42] John Preston, Jonathon Hare, Sina Samangooei, Jamie Davies, Neha Jain, and David Dupplaw. 2013. A Unified, Modular and Multimodal Approach to Search and Hyperlinking Video. In Proc. of MediaEval. Barcelona, Spain.

[43] A. Rousseau, P. Deléglise, and Y. Estève. 2014. Enhancing the TED-LIUM Corpus with Selected Data for Language Modeling And More TED Talks. In Proc. of LREC. Reykjavik, Iceland, 3935-3939.

[44] Yossi Rubner and Carlo Tomasi. 2001. Perceptual Metrics for Image Database Navigation. Springer Science \& Business Media, Norwell, MA, USA.

[45] Mathilde Sahuguet, Benoit Huet, Barbora Červenková, Evlampios Apostolidis, Vasileios Mezaris, Daniel Stein, Stefan Eickeler, José, Luis Redondo Garcia, Raphaël Troncy, and Lukáš Pikora. 2013. LinkedTV at MediaEval 2013 Search and Hyperlinking Task. In Proc. of MediaEval. Barcelona, Spain.

[46] T. Tommasi, R. Aly, K. McGuinness, K. Chatfield, R. Arandjelovic, O. Parkhi, R. J. F. Ordelman, A. Zisserman, and T. Tuytelaars. 2014. Beyond Metadata: Searching Your Archive Based on Its Audio-visual Content. In Proc. of IBC. Amsterdam, Netherlands.

[47] Steven D. Werner and Nigel G. Ward. 2013. Evaluating Prosody-Based Similarity Models for Information Retrieval. In Proc. of MediaEval. Barcelona, Spain.

[48] Frank Wilcoxon. 1945. Individual Comparisons by Ranking Methods. Biometrics Bulletin 1, 6 (1945), 80-83.

[49] Bin Xu, Weihang Liao, Zizheng Liu, Wentao Bao, Yiming Li, Daiqin Yang, Sihan Wang, Hongyi Liu, Yatong Xia, Yingbin Wang, and Zhenzheng Chen. 2015. IIPWHU@TRECVID 2015. In Proc. of TRECVID. Gaithersburg, MD, USA. 